\title{
Subband Image Coding Using Filter Banks with Non-uniform Passband Distribution
}

\author{
Xiqun Lu, Li Chen and Kwok-ping Chan \\ Department of Computer Science \\ University of Hong Kong \\ Pokfulam Road, Hong Kong
}

\begin{abstract}
In this paper, subband filter banks with nonuniform passband distribution in frequency domain are studied. Several design examples are presented and compared with conventional uniform bandwidth filter banks. Image coding results show that filter banks with non-uniform bandwidth outperform filter banks with uniform bandwidth, especially in low bit rate coding.
\end{abstract}

\section{Introduction}

In recent years, subband filter banks have been shown to be efficient in image coding applications [1][2][3]. In the analysis subsystem, an input image is decomposed into separate frequency subband sequences by the analysis filter bank. In the coding subsystem, the subband images are encoded. At the receiving end, the synthesis filter bank rebuilds an output image based on the decoded subband sequences.

The subband filter banks achieve data compression by utilizing the non-flatness of output signal spectrum and optimally allocating bit rates among the subbands based on the rate-distortion theory [1][8]. It can be shown that in terms of the spectral flatness measure [8] defined by

$\gamma_{x}^{2}=\left(1 / \sigma_{x}^{2}\right) \exp \left(1 / 2 \pi \int_{-\pi}^{+\pi} \ln S_{x}(\omega) d \omega\right)$,

the transform coding gain becomes

$$
G_{T C}=\gamma_{x}^{-2},
$$

which shows that $G_{T C}$ is the reciprocal of. the spectral flatness measure $\gamma_{x}^{2}$ of the input image $x$. Because of the non-flatness of the power-spectral density of natural images, it is expected that the coding performance increases with the number of channels [4].

Because the energy distribution of natural images is non-uniform, and the human vision system (HVS) is more sensitive to low frequency part of images [4]. Filter banks with equal bandwidth, which uniformly divide the frequency interval $[0, \pi]$, may not be the best way for image coding. The energy distribution decomposed by uniform bandwidth filter banks may not be the best "matching" to the non-flatness of the power spectral density of the input image. It is desirable to find better filter banks matched to the statistics of natural image signals.

In this paper, we attempt to design M-channel linear-phase paraunitary filter banks with nonuniform bandwidth. However, even with an optimal bandwidth, the high-order statistical dependencies of the input image can not fully be exploited by match schemes, because they would require complicated optimization procedures. In order to design non-uniform bandwidth filter banks, the ideal stopband edges of filters are to be specified. The objective function of the stopband energies of transfer functions of filters in the filter bank is optimized. As it is difficult to achieve the optimal bandwidths, the bandwidths which we give will be suboptimal.

\section{Linear-phase Paraunitary Filter Banks with Non-uniform Bandwidth}

A typical subband coding system with M-channel maximally decimated filter banks is depicted in Fig. 1, where the $H_{i}(z)$ are the analysis filters and $F_{i}(z)$ are the synthesis filters. The boxes with $\downarrow M$ denote the decimators, the boxes with $\uparrow M$ denote the expanders. Fig. 2 is a representation of the subband coding scheme in terms of the polyphase matrices, where $\mathbf{E}(z)$ is the polyphase matrix corresponding to the analysis filters, and $\mathbf{R}(z)$ is the polyphase matrix corresponding to the synthesis filters. 
In order to design a perfect reconstruction system, one way is to choose the matrix $\mathbf{E}(z)$ to be FIR paraunitary matrix, i.e.,

$$
\tilde{\mathbf{E}}(z) \mathbf{E}(z)=\mathbf{I},
$$

where $\widetilde{\mathbf{E}}(z)$ is obtained by conjugating, transposing $\mathbf{E}(z)$ and replacing $z$ by $z^{-1}$, i.e., $\widetilde{\mathbf{E}}(z)=\mathbf{E}^{T}\left(z^{-1}\right)$. The system is guaranteed to have the perfect reconstruction property by choosing $\mathbf{R}(z)=\widetilde{\mathbf{E}}(z)$ [3]. In a linear-phase paraunitary system, the polyphase matrix $\mathbf{E}(z)$ satisfies the property [1]

$$
\mathbf{E}(z)=\mathbf{D} z^{-N} \mathbf{E}\left(z^{-1}\right) \mathbf{J}_{M},
$$

where $N$ is the order of the paraunitary matrix $\mathbf{E}(z)$, the matrix $\mathbf{D}$ is a diagonal matrix whose entries are $\pm 1 ' s$. An entry +1 shows that the corresponding filter is symmetric, while an entry -1 shows that the corresponding filter is antisymmetric. The matrix $\mathbf{J}_{M}$ is a permutation matrix. It has been shown that the polyphase matrix $\mathbf{E}(z)$ is factorized into a cascade of low-order lattice building blocks

$$
\mathbf{E}(z)=\mathbf{E}_{0} \prod_{i=0}^{K-1} \mathbf{A}_{i}(z),
$$

where $\mathbf{E}_{0}$ is an initial matrix and $\mathbf{A}_{i}(z)$ is loworder factors. For filter banks with even number $(M)$ of channels, the following $\mathbf{E}_{0}$ and $\mathbf{A}_{i}(z)$ can be used [1]

$$
\begin{gathered}
\mathbf{E}_{0}=\frac{1}{\sqrt{2}}(\mathbf{V}+\mathbf{J}), \\
\mathbf{A}_{i}(z)=\mathbf{L}(z) \mathbf{Q}_{i},
\end{gathered}
$$

where

$$
\begin{gathered}
\mathbf{L}(z)=\left(\begin{array}{cc}
\mathbf{I}_{\frac{M}{2}} & \\
& z^{-1} \mathbf{I}_{\frac{M}{2}}
\end{array}\right), \\
\mathbf{Q}_{i}=\frac{1}{2}\left(\begin{array}{cc}
\mathbf{I}_{\frac{M}{2}} & \mathbf{I}_{\frac{M}{2}} \\
\mathbf{J}_{\frac{M}{2}} & -\mathbf{J}_{\frac{M}{2}}
\end{array}\right)\left(\begin{array}{ccc}
\mathbf{U}_{\frac{M}{2}} & \mathbf{0}_{\frac{M}{2}} \\
\mathbf{0}_{\frac{M}{2}} & \mathbf{V}_{\frac{M}{2}}
\end{array}\right)\left(\begin{array}{cc}
\mathbf{I}_{\frac{M}{2}} & \mathbf{J}_{\frac{M}{2}} \\
\mathbf{I}_{\frac{M}{2}} & -\mathbf{J}_{\frac{M}{2}}
\end{array}\right), \\
\mathbf{J}_{l}=\left(\begin{array}{ccc}
0 & & 1 \\
& \therefore & \\
1 & & 0
\end{array}\right)_{(l \times l)}
\end{gathered}
$$

where $\mathbf{U}_{\frac{M}{2}}$ and $\mathbf{V}_{\frac{M}{2}}$ are orthogonal matrices.

When designing filter banks with uniform bandwidth, these filters' ideal bandwidths uniformly divide the frequency interval $[0, \pi]$ into
$M$ subbands, each $\pi / M$ in width. We usually use the stopband energies of the transfer

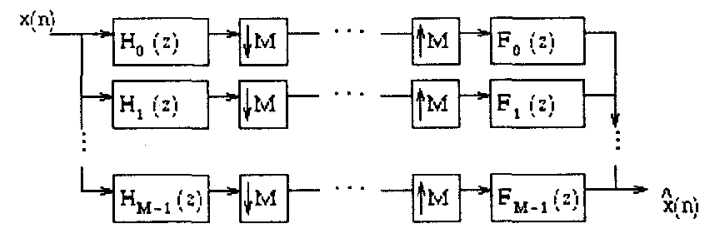

Fig. 1. A typical subband coding system with M-channel maximally decimated filter bank

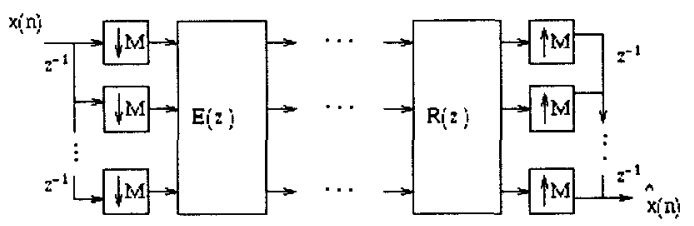

Fig. 2. Subband filters in terms of the polyphase matrices

functions of these filters as the objective function for optimization, i.e.,

$\Phi=\sum_{i=0}^{M-1}\left(\int_{0}^{\frac{i \pi}{M}}\left|H_{i}\left(e^{j \omega}\right)\right|^{2} d \omega+\int_{\frac{(i+1) \pi}{M}}^{\pi}\left|H_{i}\left(e^{j \omega}\right)\right|^{2} d \omega\right)$

In order to design non-uniform bandwidth filter banks, we first give the ideal stopband edges of filters, the objective function for optimization is adjusted as,

$$
\Phi=\sum_{i=0}^{M-1}\left(\int_{0}^{\omega_{i}}\left|H_{i}\left(e^{j \omega}\right)\right|^{2} d \omega+\int_{\omega_{i r}}^{\pi}\left|H_{i}\left(e^{j \omega}\right)\right|^{2} d \omega\right)
$$

where $\omega_{i l}$ is the $H_{i}\left(e^{j \omega}\right)$ 's left stopband edge, $\omega_{i r}$ is the $H_{i}\left(e^{j \omega}\right)$ 's right stopband edge. Now each filter's passband width is unequal, i.e. $\left|\omega_{i r}-\omega_{i l}\right| \neq\left|\omega_{j r}-\omega_{j l}\right|($ if $i \neq j)$. Although M subbands with unequal bandwidth, each subband is subsampled by $M$ in each dimension.

\section{Design Examples}

Because of the non-flatness of the power-spectral density of images, it is evident that by increasing the number of filters and decreasing their bandwidth, the radial deviation from power spectral of images can be reduced. In Fig. 3, we can see that the coding performance of an 8-channel filter bank with equal length and uniform bandwidth is better than that of a 4-channel filter bank with equal length and uniform bandwidth. All the filters are of 


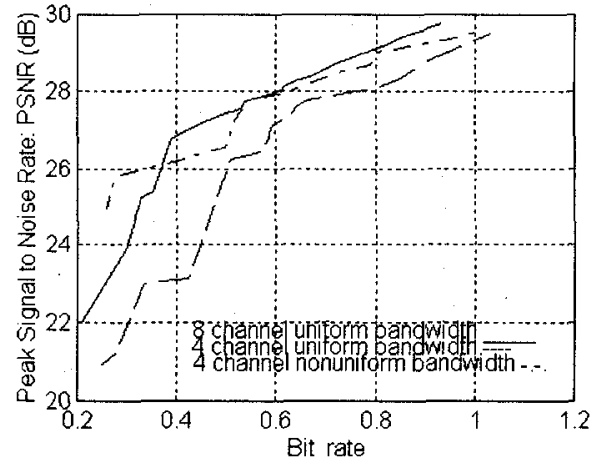

Fig. 3. Coding performances of 8-channel filter bank with uniform bandwidth, 4-channel filter bank with uniform bandwidth, 4-channel filter bank with nonuniform bandwidth

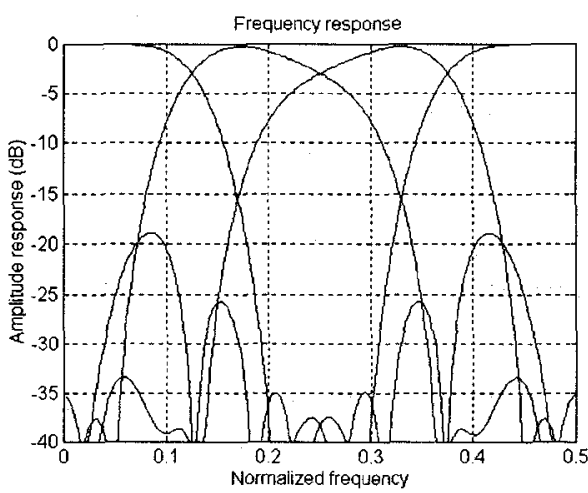

Fig. 5. 4-channel filter bank with uniform bandwidth



(a)

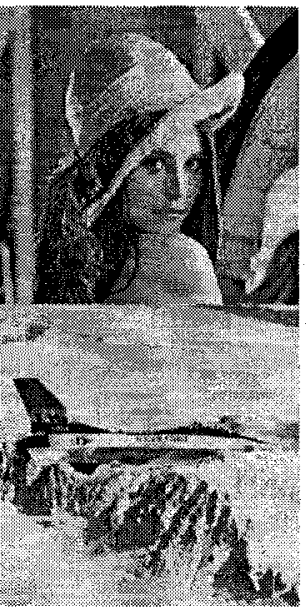

(b)

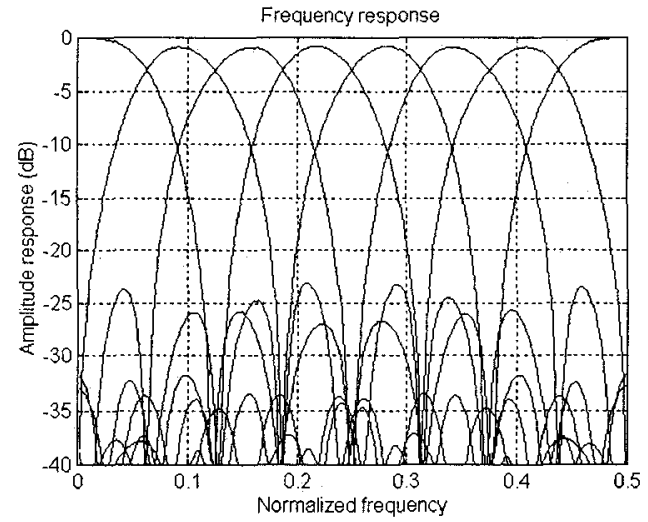

Fig. 4 Amplitude response of 8-channel filter bank with uniform bandwidth

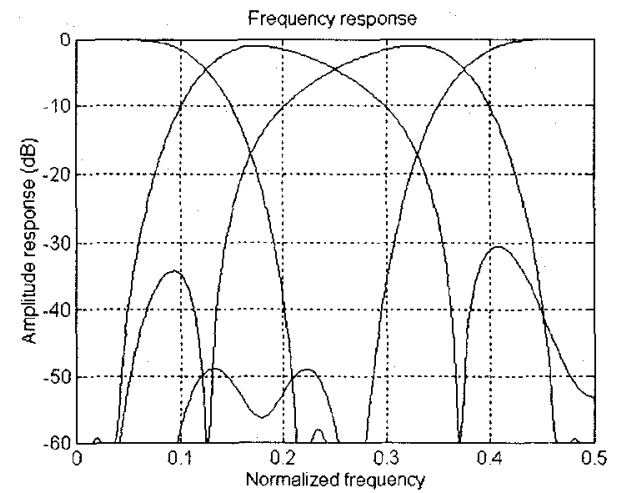

Fig. 6. 4-channel filter bank with nonuniform bandwidth

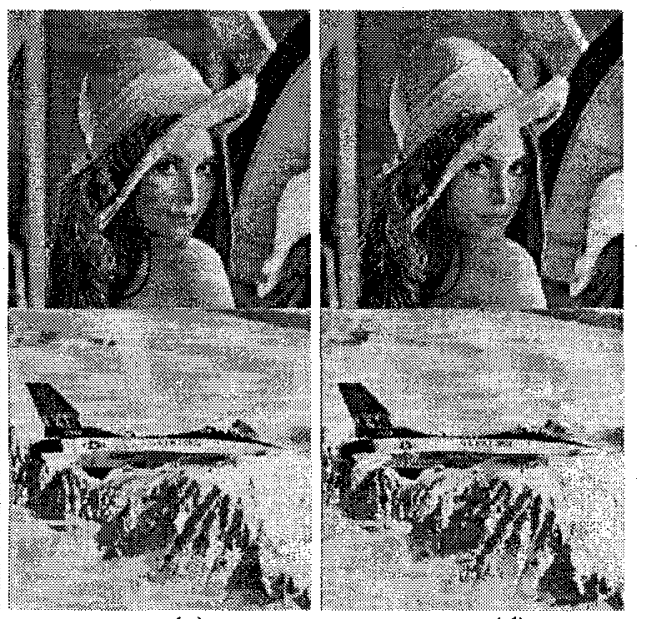

(c)

(d)

Fig. 7. (a) original images (b) 8-channel equal length uniform bandwidth (c) 4-channel filter bank equal length and uniform bandwidth (d) 4-channel filter bank with equal length but non-uniform bandwidth Table 1

\begin{tabular}{|c|c|c|c|c|c|c|}
\hline & \multicolumn{2}{|c|}{$\begin{array}{c}\text { 8-channel equal length } \\
\text { and uniform bandwidth } \\
\text { filter banks }\end{array}$} & \multicolumn{2}{|c|}{$\begin{array}{c}\text { 4-channel equal length } \\
\text { and uniform bandwidth }\end{array}$} & $\begin{array}{c}\text { 4-channel equal length } \\
\text { and non-uniform } \\
\text { bandwidth }\end{array}$ \\
\hline & bit rate & PSNR & bit rate & PSNR & bit rate & PSNR \\
\hline lenna 256 256 & 0.938 & $29.799 \mathrm{~dB}$ & 1.034 & $29.495 \mathrm{~dB}$ & 1.021 & $29.609 \mathrm{~dB}$ \\
\hline airplane 256 256 & 0.893 & $28.702 \mathrm{~dB}$ & 0.962 & $27.659 \mathrm{~dB}$ & 1.006 & $28.062 \mathrm{~dB}$ \\
\hline baboon 256 256 & 1.179 & $24.734 \mathrm{~dB}$ & 1.071 & $23.9 \mathrm{~dB}$ & 1.162 & $24.226 \mathrm{~dB}$ \\
\hline
\end{tabular}


length 24 , their amplitude responses are shown in Fig. 4, Fig. 5. But if we make little adjustment of bandwidth for each filter of 4-channel filter bank, and make it match to the energy distribution of images, the coding performance of 4-channel filter bank may be close to that of the 8-channel filter bank with uniform bandwidth. In a 4-channel 24tap filter bank with non-uniform bandwidth, we try to increase the first and second bandwidth to $0.3 \pi$ , the third and fourth bandwidth are reduced to $0.2 \pi$, the stopband edges of each filter are given by: $[0,0.3 \pi][0.3 \pi, 0.6 \pi][0.6 \pi, 0.8 \pi][0.8 \pi, \pi]$, their amplitude responses are shown in Fig. 6. At low bit rate, the coding performance of the 4channel filter bank with equal length but nonuniform bandwidth is better than that of the 8channel filter bank with equal length and uniform bandwidth as shown in Fig. 3. At high bit rate, its performance is close to that of 8-channel filter bank. In Fig. 7, the reconstructed images of these three filter banks subband coding system are presented. In Table 1, the PSNR of output images and the average bit rate allocated for images are given. In this design method, we can only make little adjustment of bandwidth for each filter in the filter banks.

\section{Conclusion}

M-channel linear-phase paraunitary filter banks with non-uniform bandwidth is reported in this paper. Several design examples are presented and compared with conventional uniform bandwidth filter banks. Image coding results show that with proper passband distribution which match the energy distribution of the input image, nonuniform bandwidth filter banks outperform filter banks with uniform bandwidth, especially in low bit rate coding.

\section{References}

[1] Soman, A.K., Vaidyanathan, P.P. and Nguyen, T.Q., "Linear phase paraunitary filter banks: theory factorizations and designs," IEEE Trans. on SP, vol. 41, pp.3480-3496, Dec. 1993.

[2] Tran, T.D. and Nguyen T.Q., "Generalized lapped orthogonal transform with unequal length basis functions," in the 1997 IEEE international Symposium on Circuits and Systems.
[3] Vaidyanathan, P.P., Multirate Systems and Filter Banks, Prentice Hall, 1993.

[4] Watson, A.B. , Ed., Digital Images and Human Vision, the MIT Press, 1993.

[5] Nguyen, T.Q. and Vaidyanathan, P.P., "Two channel perfect reconstruction FIR QMF structures which yield linear-phase analysis and synthesis filters," IEEE Trans. on ASSP, pp.676-690, May 1989.

[6] Vetterli, M. and Le Gall, D., "Perfectreconstruction filter banks: some properties and factorizations," IEEE Trans. on ASSP, pp.10571071, July 1989.

[7] Li Chen, K.P. Chan and T.Q. Nguyen, "A New Synthesis Procedure for Linear-Phase Paraunitary Digital Filter Banks," in the 1997 IEEE international Symposium on Circuits and Systems.

[8] N. S. Jayant and P. Noll, Digital Coding of Waveforms. Englewood Cliffs, N.J.: Prentice-Hall, 1984.

[9] J. Woods and S. O'neil, "Subband coding of images," IEEE Trans. on ASSP, pp.1278-1288, 1986. 